\title{
Letter to the editor of Acta Neurochirurgica: Endplate changes after lumbar discectomy with and without implantation of an annular closure device
}

\author{
Ehab Shiban ${ }^{1}$ (D) Bernhard Meyer ${ }^{1}$
}

Received: 6 May 2018 / Accepted: 1 June 2018 / Published online: 8 June 2018

(C) Springer-Verlag GmbH Austria, part of Springer Nature 2018

Dear Editor,

We have read with much interest the recent paper by Barth et al. [1]. The authors performed a post hoc analysis from the Barricade RCT looking at endplate changes (EPC) after lumbar discectomy with and without implantation of an annular closure device (ACD).

Two hundred fifty-one patients without and 242 with ACD were available for the final analysis 12 months following surgery. Baseline characteristics were similar in both groups, also with regard to number of patients with EPC. At 12-month follow-up, the number of patients with EPC and the total area of EPC (as measured on CT-scans) were significantly higher in the ACD group. These changes were considered clinically irrelevant.

The authors attributed this significant increase of EPC changes (especially in the upper endplates) to mechanical stress from the ACD on the endplates. The authors concluded: "there is a significant increase of lesion number and size within 12 months after discectomy. This increase is significantly more pronounced in the ACD group. Presence and growth of EPC is not correlated with low-back pain or ODI".

First, we would like to congratulate the authors for this well-written presentation of the data. However, we would like to propose an alternative explanation with potential long-term harm.

In the case of aseptic implant failure, most surgeons attribute spinal implant loosening to mechanical overload. In recent years, high-level evidence of the role of low virulent

This article is part of the Topical Collection on Spine - Other

Ehab Shiban

ehab.shiban@1rz.tum.de bacteria in suspected aseptic implant failure following hip and knee replacement has been reported [2]. Thereby, the role of sonication of the implants and a 14-day culture in order to prove or rule out microbiological evidence of a low-grade infection is paramount [2]. This holds true for spinal implants as well. In a prospective comparative study, implant loosening was proven as related to low-grade infections in at least half of all cases [3]. Moreover, especially in the case of ACD, we have recently reported on a symptomatic ACD loosing due to an infection by Propionibacterium acnes 5 years after implantation [4]. So this needs to be considered as a potential downside of ACDs, given the substantial colonization of some discs with low-grade bacteria.

In summary, Barth et al. describe more frequent and larger EPC changes in the ACD group. This should at least raise a high index of suspicion for potential low-grade infections. Longer follow-up is needed as these infections become symptomatic later on and may seem clinically irrelevant at 1-year follow-up.

\section{References}

1. Barth M, Weiß C, Bouma GJ, Bostelmann R, Kursumovic A, Fandino J, Thomé C (2018) Endplate changes after lumbar discectomy with and without implantation of an annular closure device. Acta Neurochir 160(4):855-862

2. Trampuz A, Piper KE, Jacobson MJ, Hanssen AD, Unni KK, Osmon DR, Mandrekar JN, Cockerill FR, Steckelberg JM, Greenleaf JF, Patel R (2007) Sonication of removed hip and knee prostheses for diagnosis of infection. N Engl J Med 357(7):654-663

3. Shiban E, Janssen I, Issa M. Meyer B. (2018) Low-grade infection in spinal instrumentation: is this the real cause of screw loosening? (Abstract: global spine congress, Singapore)

4. Lange N, Meyer B, Shiban E (2018) Symptomatic annulus-repairdevice loosening due to a low-grade infection. Acta Neurochir 160(1):199-203

1 Department of Neurosurgery, Technische Universität München, Munich, Germany 